Research Article

\title{
A Continuous-Time Model for Valuing Foreign Exchange Options
}

\author{
James J. Kung \\ School of Management, Ming Chuan University, Taipei 111, Taiwan \\ Correspondence should be addressed to James J. Kung; fnjames@mail.mcu.edu.tw
}

Received 12 February 2013; Accepted 20 May 2013

Academic Editor: Qun Lin

Copyright (C) 2013 James J. Kung. This is an open access article distributed under the Creative Commons Attribution License, which permits unrestricted use, distribution, and reproduction in any medium, provided the original work is properly cited.

\begin{abstract}
This paper makes use of stochastic calculus to develop a continuous-time model for valuing European options on foreign exchange (FX) when both domestic and foreign spot rates follow a generalized Wiener process. Using the dollar/euro exchange rate as input for parameter estimation and employing our FX option model as a yardstick, we find that the traditional Garman-Kohlhagen FX option model, which assumes constant spot rates, values incorrectly calls and puts for different values of the ratio of exchange rate to exercise price. Specifically, it undervalues calls when the ratio is between 0.70 and 1.08 , and it overvalues calls when the ratio is between 1.18 and 1.30, whereas it overvalues puts when the ratio is between 0.70 and 0.82 , and it undervalues puts when the ratio is between 0.86 and 1.30 .
\end{abstract}

\section{Introduction}

In this paper, we make use of stochastic calculus [1-3] to develop a continuous-time model for valuing European options on foreign exchange when both domestic and foreign spot rates follow a generalized Wiener process.

Foreign exchange (FX) options have traded on the Philadelphia Stock Exchange since 1982. An FX option is an agreement between two parties in which one party pays a premium and obtains the right to buy or sell the stated amount of foreign exchange at a later date at the exercise price, where the exercise price is an exchange rate agreed upon at the initial time. Extending the Black-Scholes [4] pricing model for stock options and assuming that both domestic and foreign spot rates are constant during the life of the option, Garman and Kohlhagen [5] developed in 1983 a model for valuing European FX options.

However, the assumption of Garman and Kohlhagen (G-K) that the two spot rates are constant over the life of the option is inappropriate because they are, in actuality, evolving continuously and stochastically through time. In this study, we incorporate the stochastic character of the two spot rates into our FX option model. Specifically, we employ the following stochastic process, often referred to as the generalized Wiener process, to represent the evolution of the spot rate $r(t)$ and, accordingly, derive a continuous-time model for valuing European call and put FX options as

$$
d r(t)=a d t+b d W .
$$

In (1), the generalized Wiener process has a drift rate of $a$, a variance rate of $b^{2}$, and $W$ is a standard Wiener process whose increment $d W$ has a normal distribution with zero mean and variance $d t$.

Remark 1. It is possible that the spot rate under (1) can become negative. But negative spot rate is not probable if the drift rate is positive. More importantly, most FX options traded on an exchange have an expiration of less than one year. Hence, if we use an initial positive value for $r(t)$ and suitable values for the drift rate and variance rate, the expected first-passage time of the spot rate to the origin can easily be made longer than one year.

Remark 2. Our FX option model essentially extends the traditional G-K model to incorporate the stochastic character of the two spot rates. Like the G-K model, our model is for valuing European FX options. To value American options, often we have to resort to numerical procedures because no analytic formulas are available. For some well-articulated 
numerical procedures for valuing American options, see Hull [6] for pricing FX options with constant interest rates, Ho et al. [7] for pricing stock options with stochastic interest rates, and Zhang and Wang [8] for pricing bond options with a penalty method (see $[9,10])$.

The rest of this paper is organized as follows. In Section 2, we derive an explicit formula for valuing European call and put FX options when both domestic and foreign spot rates evolve according to (1). In Section 3, we first estimate the various parameters of our FX option model based on the dollar/euro exchange rate; then, we compute the FX option prices for our model and the G-K model using the parameter estimates as inputs, and finally we examine the pricing biases in the $\mathrm{G}-\mathrm{K}$ model employing our model as a yardstick. A short conclusion is given in Section 4. The derivation of the rather lengthy equation (27) in Section 2 is relegated to the Appendix.

\section{Deriving a Formula for Call and Put FX Options}

In this paper, we assume that the foreign exchange market is frictionless; that is, there are no trading costs, margin requirements, exchange rate controls, and taxes; trading takes place continuously; borrowing and short-selling are allowed; there exist pure discount bonds at which each currency can be borrowed or lent. To proceed, we adopt the following notation:

$S(t)$ : the spot exchange rate (domestic currency price per unit of foreign currency) at time $t$,

$r_{d}(t)$ : the domestic spot rate of interest at time $t$,

$r_{f}(t)$ : the foreign spot rate of interest at time $t$,

$B_{d}(t, T)$ : the domestic currency price of a pure discount bond at time $t$ which pays one unit of domestic currency at time $t+T$,

$B_{f}(t, T)$ : the foreign currency price of a pure discount bond at time $t$ which pays one unit of foreign currency at time $t+T$

$c(t, T)$ : the domestic currency price at time $t$ of a European call on one unit of foreign currency which expires at time $t+T$,

$p(t, T)$ : the domestic currency price at time $t$ of a European put on one unit of foreign currency which expires at time $t+T$,

$X$ : the domestic currency exercise price of a European call or a put on foreign currency.

Using (1) for the domestic and foreign spot rates, their diffusion processes are expressed as follows:

$$
\begin{aligned}
& d r_{d}(t)=a_{d} d t+b_{d} d W_{d}, \\
& d r_{f}(t)=a_{f} d t+b_{f} d W_{f},
\end{aligned}
$$

with $d W_{d} d W_{f}=\rho_{d f} d t$. Using (2) and applying Ito's lemma [11-13], we have

$$
\begin{aligned}
& d B_{d}=\left[\frac{\partial B_{d}}{\partial t}+a_{d} \frac{\partial B_{d}}{\partial r_{d}}+\frac{1}{2} b_{d}^{2} \frac{\partial^{2} B_{d}}{\partial r_{d}^{2}}\right] d t+b_{d} \frac{\partial B_{d}}{\partial r_{d}} d W_{d} \\
& d B_{f}=\left[\frac{\partial B_{f}}{\partial t}+a_{f} \frac{\partial B_{f}}{\partial r_{f}}+\frac{1}{2} b_{f}^{2} \frac{\partial^{2} B_{f}}{\partial r_{f}^{2}}\right] d t+b_{f} \frac{\partial B_{f}}{\partial r_{f}} d W_{f} .
\end{aligned}
$$

Letting

$$
\begin{gathered}
\mu_{d}=\frac{1}{B_{d}}\left[\frac{\partial B_{d}}{\partial t}+a_{d} \frac{\partial B_{d}}{\partial r_{d}}+\frac{1}{2} b_{d}^{2} \frac{\partial^{2} B_{d}}{\partial r_{d}^{2}}\right], \\
\sigma_{d}=\frac{-1}{B_{d}}\left[b_{d} \frac{\partial B_{d}}{\partial r_{d}}\right]=\frac{-b_{d}}{B_{d}} \frac{\partial B_{d}}{\partial r_{d}}, \\
\mu_{f}=\frac{1}{B_{f}}\left[\frac{\partial B_{f}}{\partial t}+a_{f} \frac{\partial B_{f}}{\partial r_{f}}+\frac{1}{2} b_{f}^{2} \frac{\partial^{2} B_{f}}{\partial r_{f}^{2}}\right], \\
\sigma_{f}=\frac{-1}{B_{f}}\left[b_{f} \frac{\partial B_{f}}{\partial r_{f}}\right]=\frac{-b_{f}}{B_{f}} \frac{\partial B_{f}}{\partial r_{f}},
\end{gathered}
$$

and assuming the local expectations hypothesis holds for the term structure of interest rates (i.e., $\mu_{d}=r_{d}$ and $\mu_{f}=r_{f}$ ), we obtain

$$
\begin{aligned}
& \frac{d B_{d}}{B_{d}}=r_{d} d t+\sigma_{d} d W_{d}, \\
& \frac{d B_{f}}{B_{f}}=r_{f} d t+\sigma_{f} d W_{f} .
\end{aligned}
$$

Solving (5) and (6) for $B_{d}$ and $B_{f}$, we obtain explicit formulas for the prices of domestic and foreign pure discount bonds with time to maturity $T$ as

$$
\begin{aligned}
& B_{d}(t, T)=\exp \left\{-r_{d} T-\frac{a_{d} T^{2}}{2}+\frac{b_{d}^{2} T^{3}}{6}\right\}, \\
& B_{f}(t, T)=\exp \left\{-r_{f} T-\frac{a_{f} T^{2}}{2}+\frac{b_{f}^{2} T^{3}}{6}\right\} .
\end{aligned}
$$

Note that $T=-\left(1 / B_{d}\right)\left[\partial B_{d} / \partial r_{d}\right]=-\left(1 / B_{f}\right)\left[\partial B_{f} / \partial r_{f}\right]$. Hence, we have $\sigma_{d}=-\left(b_{d} / B_{d}\right)\left[\partial B_{d} / \partial r_{d}\right]=b_{d} T$ and $\sigma_{f}=$ $-\left(b_{f} / B_{f}\right)\left[\partial B_{f} / \partial r_{f}\right]=b_{f} T$.

Similar to the G-K FX option model, we assume that the spot exchange rate follows the geometric Wiener process

$$
\frac{d S}{S}=\mu_{s} d t+\sigma_{s} d W_{s},
$$

where $\mu_{s}$ and $\sigma_{s}$ are constant parameters, and $W_{s}$ is a standard Wiener process. In addition, we assume $d W_{s} d W_{d}=\rho_{s d} d t$ and $d W_{s} d W_{f}=\rho_{s f} d t$.

Converting the price of a foreign pure discount bond into domestic currency price, we define a new variable $G=S B_{f}$ such that

$$
\frac{d G}{G}=\mu_{G} d t+\sigma_{G} d W_{G}
$$


Applying Ito's lemma to the call function $c \equiv c(t, T) \equiv$ $c\left(G, B_{d}, T ; X\right)$ and the relation $d t=-d T$, we obtain

$$
\begin{aligned}
d c= & \frac{\partial c}{\partial G} d G+\frac{\partial c}{\partial B_{d}} d B_{d} \\
- & {\left[\frac{1}{2} \frac{\partial^{2} c}{\partial G^{2}} \sigma_{G}^{2} G^{2}+\frac{1}{2} \frac{\partial^{2} c}{\partial B_{d}^{2}} \sigma_{d}^{2} B_{d}^{2}\right.} \\
& \left.+\frac{\partial^{2} c}{\partial G \partial B_{d}} \rho_{G d} \sigma_{G} \sigma_{d} G B_{d}-\frac{\partial c}{\partial T}\right] d T .
\end{aligned}
$$

At this point, we set up a hedge consisting of three assets: $G, B_{d}$, and $c$. Let $Q_{G}$ be the number of $G, Q_{d}$ the number of $B_{d}$, and $Q_{c}$ the number of $c$ in the hedge. Let $P_{h}$ be the value of the hedge portfolio. The hedge is set up in such a way that the value of this hedge portfolio is zero, that is, $P_{h}=Q_{G} G+$ $Q_{d} B_{d}+Q_{c} c=0$. Hence, the change in the value of this hedge portfolio is also zero, that is,

$$
d P_{h}=Q_{G} d G+Q_{d} d B_{d}+Q_{c} d c=0 .
$$

Remark 3. Our hedge is different from that of Black and Scholes [4]. In their case, they create their hedge such that the hedge portfolio is riskless. Hence, its return must equal the riskless rate or the spot rate. In our case, we create our hedge such that the value $P_{h}$ of the hedge portfolio is zero (i.e., the aggregate investment is zero). Hence, we have $d P_{h}=0$ in (11).

Substituting (5), (9), and (10) into (11) and grouping, (11) becomes

$$
\begin{aligned}
d P_{h}=Q_{c}\left[\frac{1}{2} \frac{\partial^{2} c}{\partial G^{2}} \sigma_{G}^{2} G^{2}+\frac{1}{2} \frac{\partial^{2} c}{\partial B_{d}^{2}} \sigma_{d}^{2} B_{d}^{2}\right. \\
\left.+\frac{\partial^{2} c}{\partial G \partial B_{d}} \rho_{G d} \sigma_{G} \sigma_{d} G B_{d}-\frac{\partial c}{\partial T}\right] d T \\
+\left[Q_{c} \frac{\partial c}{\partial G}+Q_{G}\right] d G+\left[Q_{c} \frac{\partial c}{\partial B_{d}}+Q_{d}\right] d B_{d}=0
\end{aligned}
$$

Equation (12) suggests that $Q_{c}(\partial c / \partial G)+Q_{G}=0, Q_{c}(\partial c /$ $\left.\partial B_{d}\right)+Q_{d}=0$, and

$$
\frac{1}{2} \frac{\partial^{2} c}{\partial G^{2}} \sigma_{G}^{2} G^{2}+\frac{1}{2} \frac{\partial^{2} c}{\partial B_{d}^{2}} \sigma_{d}^{2} B_{d}^{2}+\frac{\partial^{2} c}{\partial G \partial B_{d}} \rho_{G d} \sigma_{G} \sigma_{d} G B_{d}-\frac{\partial c}{\partial T}=0
$$

Then the price of a call must satisfy (13) subject to two boundary conditions: (i) the call price is zero if $G=0$; (ii) at its expiration, the call has a value of either zero if $G \leq X$ or $G-X$ if $G>X$. In notations, the two boundary conditions are

$$
\begin{gathered}
c\left(G=0, B_{d}, T ; X\right)=0, \\
c\left(G, B_{d}=1,0 ; X\right)=\max (0, G-X) .
\end{gathered}
$$

The second-order partial differential equation in (13) has no well-known solution. Hence, to solve for $c$ in (13), we transform (13) to a standard heat equation [14-16] of the form $u_{t}(x, t)=\alpha^{2} u_{x x}(x, t)$, where $\alpha$ is some constant. Making use of the linear homogeneity of $c$ in $G$ and $X B_{d}$, we can carry out such transformation for (13). Consequently, we set $\theta \equiv \theta\left(G, B_{d}, T\right)=G / X B_{d}$.

Remark 4. A function $f \equiv f\left(x_{1}, \ldots, x_{n}\right)$ is said to be linear homogeneous or homogeneous of degree one in $x_{i}$, where $i=$ $1, \ldots, n$, if $f\left(\alpha x_{1}, \ldots, \alpha x_{n}\right)=\alpha f\left(x_{1}, \ldots, x_{n}\right)$, where $\alpha$ is some constant. In a competitive and perfect market, the fact that the value of a call is homogeneous of degree one in the asset and exercise pricemeans that the value of the call with exercise price $X$ when the asset value is $G$ will be exactly $\alpha$ times the value of a call on the same asset with exercise price $X / \alpha$ when the asset value is $G / \alpha$. See [17-19] for more description on linear homogeneity.

Using Ito's lemma and (5) and (9), the total differential of $\theta$ is given by

$$
\begin{aligned}
d \theta= & \frac{\partial \theta}{\partial G} \mu_{G} G+\frac{\partial \theta}{\partial B_{d}} r_{d} B_{d}+\frac{\partial \theta}{\partial t}+\frac{1}{2} \frac{\partial^{2} \theta}{\partial G^{2}} \sigma_{G}^{2} G^{2} \\
& \left.+\frac{1}{2} \frac{\partial^{2} \theta}{\partial B_{d}^{2}} \sigma_{d}^{2} B_{d}^{2}+\frac{\partial^{2} \theta}{\partial G \partial B_{d}} \rho_{G d} \sigma_{G} \sigma_{d} G B_{d}\right] d t \\
+ & \frac{\partial \theta}{\partial G} \sigma_{G} G d W_{G}+\frac{\partial \theta}{\partial B_{d}} \sigma_{d} B_{d} d W_{d} .
\end{aligned}
$$

Substituting $\partial \theta / \partial G=1 / X B_{d}, \partial \theta / \partial B_{d}=-G / X B_{d}^{2}, \partial \theta / \partial t=$ $0, \partial^{2} \theta / \partial G^{2}=0, \partial^{2} \theta / \partial B_{d}^{2}=G / X B_{d}^{3}$, and $\partial^{2} \theta / \partial G \partial B_{d}=-1 / X B_{d}^{2}$ into (15) and simplifying, (15) becomes

$$
\frac{d \theta}{\theta}=\mu_{\theta} d t+\sigma_{\theta} d W_{\theta}
$$

where $\mu_{\theta}=\mu_{G}-r_{d}+\left(\sigma_{d}^{2} / 2\right)-\rho_{G d} \sigma_{G} \sigma_{d}$ and $\sigma_{\theta}^{2}=\sigma_{G}^{2}+\sigma_{d}^{2}-$ $2 \rho_{G d} \sigma_{G} \sigma_{d}$.

To solve (13) for $c\left(G, B_{d}, T ; X\right)$ subject to the two boundary conditions in (14), we use another variable $K$ such that $K \equiv K(\theta, T ; X)=\left(c\left(G, B_{d}, T ; X\right)\right) / X B_{d}$. In words, $K$ is the call price expressed in the same units as $\theta$. Expressed in another way, $c\left(G, B_{d}, T ; X\right)=X B_{d} K(\theta, T ; X)$. Then $\partial^{2} c / \partial G^{2}=\left(1 / X B_{d}\right)\left(\partial^{2} K / \partial G^{2}\right), \partial^{2} c / \partial B_{d}^{2}=\left(G^{2} / X B_{d}^{3}\right)\left(\partial^{2} K /\right.$ $\left.\partial G^{2}\right), \partial^{2} c / \partial G \partial B_{d}=-\left(G / X B_{d}^{2}\right)\left(\partial^{2} K / \partial G^{2}\right)$, and $\partial c / \partial T=$ $X B_{d}(\partial K / \partial T)$. Substituting them into (13) and simplifying, (13) becomes

$$
\frac{1}{2}\left(\frac{G^{2}}{X B_{d}}\right)\left[\sigma_{G}^{2}+\sigma_{d}^{2}-2 \rho_{G d} \sigma_{G} \sigma_{d}\right] \frac{\partial^{2} K}{\partial G^{2}}-X B_{d} \frac{\partial K}{\partial T}=0,
$$

or

$$
\frac{1}{2}\left(\frac{G}{X B_{d}}\right)^{2}\left[\sigma_{G}^{2}+\sigma_{d}^{2}-2 \rho_{G d} \sigma_{G} \sigma_{d}\right] \frac{\partial^{2} K}{\partial G^{2}}-\frac{\partial K}{\partial T}=0 .
$$

$$
\text { Since } \theta^{2}=\left(G / X B_{d}\right)^{2} \text { and } \sigma_{\theta}^{2}=\sigma_{G}^{2}+\sigma_{d}^{2}-2 \rho_{G d} \sigma_{G} \sigma_{d} \text {, }
$$

$$
\frac{1}{2} \sigma_{\theta}^{2} \theta^{2} \frac{\partial^{2} K}{\partial \theta^{2}}-\frac{\partial K}{\partial T}=0
$$


In other words, $K \equiv K(\theta, T ; X)$ has to satisfy (19) subject to the following two boundary conditions: $K(\theta=0, T ; X)=0$ and $K(\theta, 0 ; X)=\max (0, \theta-1)$.

Remark 5. Given $\theta=G / X B_{d}, G=0$ is equivalent to $\theta=0$. Thus, we have the first boundary condition $K(\theta=0, T ; X)=$ 0 . At time $t+T, B_{d}(T, T)=1$, and we have the second boundary condition $K(\theta, 0 ; X)=\left(c\left(G, B_{d}(T, T), 0 ; X\right)\right) /\left(X B_{d}(T, T)\right)$ $=(\max (0, G-X)) / X=\max (0,(G / X)-1)=\max (0, \theta-1)$.

Setting up another variable $V \equiv \int_{t}^{t+T} \sigma_{\theta}^{2}(v) d v$ and then defining $(\theta, V) \equiv K(\theta, T)$, we have that $\partial^{2} K / \partial \theta^{2}=\partial^{2} y / \partial \theta^{2}$ and $\partial K / \partial T=(\partial y / \partial V)(\partial V / \partial T)=(\partial y / \partial V) \sigma_{\theta}^{2}$. Substituting them into (19), we obtain

$$
\frac{1}{2} \sigma_{\theta}^{2} \theta^{2} \frac{\partial^{2} y}{\partial \theta^{2}}-\frac{\partial y}{\partial V} \sigma_{\theta}^{2}=\sigma_{\theta}^{2}\left[\frac{1}{2} \theta^{2} \frac{\partial^{2} y}{\partial \theta^{2}}-\frac{\partial y}{\partial V}\right]=0
$$

or

$$
\frac{1}{2} \theta^{2} \frac{\partial^{2} y}{\partial \theta^{2}}-\frac{\partial y}{\partial V}=0
$$

In other words, $y=y(\theta, V)$ has to satisfy (21) subject to the following two boundary conditions: $y(0, V)=0$ and $y(\theta, 0)=$ $\max (0, \theta-1)$.

To solve (21) subject to the two boundary conditions, we transform (21) by using the change of variables $\delta \equiv \log \theta+$ $(V / 2)$ and $u(\delta, V) \equiv y(\theta, V) / \theta$. Then we have $y(\theta, V)=$ $u\left(\log \theta+(1 / 2) \int_{t}^{t+T} \sigma_{\theta}^{2}(v) d v, \int_{t}^{t+T} \sigma_{\theta}^{2}(v) d v\right) \theta, \partial^{2} y / \partial \theta^{2}=$ $(1 / \theta)\left((\partial u / \partial \delta)+\left(\partial^{2} u / \partial \delta^{2}\right)\right)$, and $\partial y / \partial V=\theta((1 / 2)(\partial u / \partial \delta)+$ $(\partial u / \partial V))$. Substituting them into (21) and simplifying, (21) becomes $\partial u / \partial V=(1 / 2)\left(\partial^{2} u / \partial \delta^{2}\right)$. For the first boundary condition, we have that $u(\delta, V)=y(\theta, V) / \theta=(1 / \theta) K(\theta, T)=$ $(1 / \theta)\left(c / X B_{d}\right)=\left(X B_{d} / G\right)\left(c / X B_{d}\right)=c / G$. As always, $0 \leq c \leq$ $G$. Thus, the first boundary condition is $|u(\delta, V)|=(c / G) \leq 1$. For the second boundary condition, we have that $1-(1 / \theta)=$ $1-\exp [-\log \theta]=1-\exp [-\delta]$, because $\delta=\log \theta$ when $V=0$. Hence, $u(\delta, V=0)=(\theta-1) / \theta=1-(1 / \theta)=1-\exp [-\delta]$ if $1 \geq \exp [-\delta]$ and $u(\delta, V=0)=0$ if $1<\exp [-\delta]$. In short, our boundary value problem is made up of a boundedness condition $|u(\delta, V)| \leq 1$ and the following two conditions:

$$
\begin{aligned}
\frac{\partial u(\delta, V)}{\partial V} & =\frac{1}{2} \frac{\partial^{2} u(\delta, V)}{\partial \delta^{2}}, \\
u(\delta, 0) & = \begin{cases}1-\exp [-\delta] & \text { if } 1 \geq \exp [-\delta] \\
0 & \text { if } 1<\exp [-\delta]\end{cases}
\end{aligned}
$$

Now (22) is in standard heat equation form and hence can be solved by the separation-of-variables method. Let $u(\delta, V)=f(\delta) g(V)$, where $f(\delta)$ is some function of $\delta$ and $g(V)$ is some function of $V$. Substituting $u(\delta, V)=f(\delta) g(V)$ into (22) and simplifying, we get

$$
\frac{2}{g(V)} \frac{\partial g(V)}{\partial V}=\frac{1}{f(\delta)} \frac{\partial^{2} f(\delta)}{\partial \delta^{2}}
$$

In other words, the right-hand side of (24) depends only on $\delta$ and the left-hand side depends only on $V$. Since $\delta$ and $V$ are independent variables, we can equate the two sides of (24) to a constant $-k^{2}$, where $k>0$.

Remark 6. We equate the two sides of (24) to $-k^{2}$ so that the two differential equations in (25) have continuous eigenvalues $k^{2}$.

Hence, by setting the two sides of (24) equal to $-k^{2}$, we obtain the following two ordinary differential equations:

$$
\begin{aligned}
& \frac{\partial g(V)}{\partial V}+\frac{1}{2} k^{2} g(V)=0, \\
& \frac{\partial^{2} f(\delta)}{\partial \delta^{2}}+k^{2} f(\delta)=0 .
\end{aligned}
$$

Rewriting (25), we have $g(V)=\exp \left(-(1 / 2) k^{2} V\right)$ and $f(\delta)=A(\delta) \cos (k \delta)+B(\delta) \sin (k \delta)$. The generalized linear combination of functions $u(\delta, V)=f(\delta) g(V)$ becomes

$$
\begin{aligned}
u(\delta, V)=\int_{0}^{\infty} & {[A(k) \cos (k \delta)+B(k) \sin (k \delta)] } \\
& \times \exp \left(-\frac{1}{2} k^{2} V\right) d k
\end{aligned}
$$

By the Fourier integral theorem, the expression in (26) is legitimate if $A(k)=(1 / \pi) \int_{-\infty}^{\infty} f(\omega) \cos (k \omega) d \omega$ and $B(k)=$ $(1 / \pi) \int_{-\infty}^{\infty} f(\omega) \sin (k \omega) d \omega$. Substituting $A(k)$ and $B(k)$ into (26), we obtain (see the Appendix for derivation of (27))

$$
\begin{gathered}
u(\delta, V)=\frac{1}{\pi} \int_{0}^{\infty}\left\{\int_{-\infty}^{\infty}[\cos (k \omega) \cos (k \delta)+\sin (k \omega) \sin (k \delta)]\right. \\
\quad \times f(\omega) d \omega\} \exp \left(-\frac{1}{2} k^{2} V\right) d k \\
=\frac{1}{\sqrt{2 V \pi}} \int_{-\infty}^{\infty} f(\omega) \exp \left(-\frac{(\omega-\delta)^{2}}{2 V}\right) d \omega .
\end{gathered}
$$

Setting $q=(\omega-\delta) / \sqrt{2 V}$, we have $\omega=\delta+q \sqrt{2 V}$ and $d \omega=\sqrt{2 V} d q$. Substituting $q=(\omega-\delta) / \sqrt{2 V}$ and (24) into (27), we get

$$
\begin{aligned}
u(\delta, V)= & \frac{1}{\sqrt{\pi}} \int_{-\infty}^{\infty} f(\delta+q \sqrt{2 V}) \exp \left(-q^{2}\right) d q \\
= & \frac{1}{\sqrt{\pi}} \int_{-\delta / \sqrt{2 V}}^{\infty}[1-\exp (-\delta-q \sqrt{2 V})] \exp \left(-q^{2}\right) d q \\
= & \frac{1}{\sqrt{\pi}} \int_{-\delta / \sqrt{2 V}}^{\infty} \exp \left(-q^{2}\right) d q \\
& -\frac{1}{\sqrt{\pi}} \int_{-\delta / \sqrt{2 V}}^{\infty} \exp \left(-\delta-q \sqrt{2 V}-q^{2}\right) d q .
\end{aligned}
$$


In order to solve (28), we make another change of variables by setting $a=\sqrt{2} q$. Then the first term of (28) becomes

$$
\begin{aligned}
\frac{1}{\sqrt{\pi}} \int_{-\delta / \sqrt{2 V}}^{\infty} \exp \left(-q^{2}\right) d q & =\frac{1}{\sqrt{2 \pi}} \int_{-\delta / \sqrt{V}}^{\infty} \exp \left(-\frac{a^{2}}{2}\right) d a \\
& =\frac{1}{\sqrt{2 \pi}} \int_{-\infty}^{\delta / \sqrt{V}} \exp \left(-\frac{a^{2}}{2}\right) d a \\
& =N\left(\frac{\delta}{\sqrt{V}}\right)=N\left(a_{1}\right),
\end{aligned}
$$

where $a_{1}=\delta / \sqrt{V}$ and $N(\cdot)$ is the cumulative probability distribution function for a standardized normal random variable; that is, $N\left(a_{1}\right)$ is the probability that such a variable will be less than $a_{1}$.

For the second term of (28), note that $-\delta-q \sqrt{2 V}-q^{2}=$ $-\log \theta-(V / 2)-a \sqrt{V}-\left(a^{2} / 2\right)=\log (1 / \theta)-(1 / 2)\left[a^{2}+2 a \sqrt{V}+\right.$ $\left.(\sqrt{V})^{2}\right]=\log (1 / \theta)-(1 / 2)(a+\sqrt{V})^{2}$. Hence, the integrand of the second term is $\exp \left(-\delta-q \sqrt{2 V}-q^{2}\right)=\exp [\log (1 / \theta)-$ $\left.(1 / 2)(a+\sqrt{V})^{2}\right]=(1 / \theta) \exp \left[-(1 / 2)(a+\sqrt{V})^{2}\right]$. Substituting it into the second term and simplifying, (28) becomes

$$
\begin{aligned}
& \frac{1}{\sqrt{\pi}} \int_{-\delta / \sqrt{2 V}}^{\infty} \exp \left(-\delta-q \sqrt{2 V}-q^{2}\right) d q \\
& \quad=\frac{1}{\theta \sqrt{2 \pi}} \int_{-\infty}^{a_{1}} \exp \left[-\frac{1}{2}(a+\sqrt{V})^{2}\right] d a \\
& \quad=\frac{1}{\theta \sqrt{2 \pi}} \int_{-\infty}^{a_{2}} \exp \left[-\frac{b^{2}}{2}\right] d b=\frac{1}{\theta} N\left(a_{2}\right),
\end{aligned}
$$

where $b=a+\sqrt{V}$ and $a_{2}=a_{1}-\sqrt{V}$. Combining (29) and (30), we obtain

$$
u(\delta, V)=N\left(a_{1}\right)-\frac{1}{\theta} N\left(a_{2}\right),
$$

or

$$
y(\theta, V)=\theta u(\delta, V)=\theta N\left(a_{1}\right)-N\left(a_{2}\right) .
$$

As stated earlier, $G=S B_{f}, \theta=G / X B_{d}$, and $c\left(G, B_{d}\right.$, $T ; X)=X B_{d} K(\theta, T ; X)=X B_{d} y(\theta, V)$. By the linear homogeneity property of $c \equiv c(t, T) \equiv c\left(G, B_{d}, T ; X\right)$, the price of a European call FX option is

$$
c=X B_{d}\left[\theta N\left(a_{1}\right)-N\left(a_{2}\right)\right]=S B_{f} N\left(a_{1}\right)-X B_{d} N\left(a_{2}\right),
$$

where $a_{1}=\delta / \sqrt{V}=(\log \theta+(V / 2)) / \sqrt{V}$ and $a_{2}=a_{1}-\sqrt{V}=$ $(\log \theta-(V / 2)) / \sqrt{V}$. In addition, $V$ is given by

$$
\begin{aligned}
V \equiv & \int_{t}^{t+T} \sigma_{\theta}^{2}(v) d v \\
= & \int_{t}^{t+T}\left[\sigma_{s}^{2}-2\left(\rho_{s d} \sigma_{s} b_{d}-\rho_{s f} \sigma_{s} b_{f}\right) v\right. \\
& \left.\quad+\left(b_{d}^{2}+b_{f}^{2}-2 \rho_{d f} b_{d} b_{f}\right) v^{2}\right] d v \\
= & \sigma_{s}^{2} T-\left(\rho_{s d} \sigma_{s} b_{d}-\rho_{s f} \sigma_{s} b_{f}\right) T^{2} \\
& +\frac{1}{3}\left(b_{d}^{2}+b_{f}^{2}-2 \rho_{d f} b_{d} b_{f}\right) T^{3} .
\end{aligned}
$$

According to put-call parity for European options, the price $p \equiv p\left(G, B_{d}, T ; X\right)$ of a European put FX option is

$$
p=c-S B_{f}+X B_{d}=X B_{d} N\left(-a_{2}\right)-S B_{f} N\left(-a_{1}\right) .
$$

Equations (33) through (35), as a whole, constitute our FX option model. If both domestic and foreign spot rates are constant (i.e., $a_{d}=a_{f}=b_{d}=b_{f}=0$ in (2)), then the domestic and foreign bond prices in (7) become $B_{d}(t, T)=\exp \left(-r_{d} T\right)$ and $B_{f}(t, T)=\exp \left(-r_{f} T\right)$, and $V \equiv \int_{t}^{t+T} \sigma_{\theta}^{2}(v) d v=\sigma_{s}^{2} T$. Substituting them into (33) and (35), our FX model then reduces to the following G-K FX model for valuing European call and put options:

$$
\begin{gathered}
c_{G K}=S \exp \left(-r_{f} T\right) N\left(d_{1}\right)-X \exp \left(-r_{d} T\right) N\left(d_{2}\right), \\
p_{G K}=X \exp \left(-r_{d} T\right) N\left(-d_{2}\right)-S \exp \left(-r_{f} T\right) N\left(-d_{1}\right),
\end{gathered}
$$

where $d_{1}=\left(\log (S / X)+\left(r_{d}-r_{f}+\sigma_{s}^{2} / 2\right) T\right) / \sigma_{s} \sqrt{T}$ and $d_{2}=$ $d_{1}-\sigma_{s} \sqrt{T}$.

\section{FX Option Prices and Pricing Biases in the G-K Model}

Retrieved from the Datastream database, three sets of daily data (a total of 2,869 observations from 4 January 1999 to 31 December 2009) are used for parameter estimation. One set is the dollar/euro exchange rate and the other two sets are the 3month US. Treasury Bill Rate and the 1-month euro-currency rate; that is, we use the Treasury Bill Rate to represent the domestic spot rate $r_{d}$ and the euro-currency rate to represent the foreign spot rate $r_{f}$. Accordingly, the estimates for the six parameters of our FX option model are as follows: $\widehat{\sigma_{s}}=$ $0.198428, \widehat{b_{d}}=0.018534, \widehat{b_{f}}=0.011398, \widehat{\rho_{s d}}=-0.347350, \widehat{\rho_{s f}}=$ -0.297520 , and $\widehat{\rho_{d f}}=0.524996$.

Remark 7. The euro was introduced as an accounting currency on 1 January 1999. Euro coins and banknotes have been in circulation since 1 January 2002.

Using the above parameter estimates as inputs, we compute the option prices for our FX model ((33)-(35)) and 
TABLE 1: Prices for European call and put FX options when time to maturity $T=1$ month.

\begin{tabular}{|c|c|c|c|c|c|c|}
\hline$S_{t} / X$ & Call $_{O}$ & Call $_{\mathrm{GK}}$ & Bias $_{c}$ & $\mathrm{Put}_{\mathrm{O}}$ & $\mathrm{Put}_{\mathrm{GK}}$ & Bias $_{p}$ \\
\hline 0.70 & 0.000000 & 0.000000 & & 0.617117 & 0.617122 & -0.0008 \\
\hline 0.72 & 0.000000 & 0.000000 & & 0.560023 & 0.560026 & -0.0005 \\
\hline 0.74 & 0.000000 & 0.000000 & & 0.506014 & 0.506017 & -0.0006 \\
\hline 0.76 & 0.000000 & 0.000000 & & 0.454848 & 0.454851 & -0.0007 \\
\hline 0.78 & 0.000000 & 0.000000 & & 0.406307 & 0.406308 & -0.0002 \\
\hline 0.80 & 0.000001 & 0.000001 & & 0.360193 & 0.360194 & -0.0003 \\
\hline 0.82 & 0.000006 & 0.000006 & & 0.316332 & 0.316333 & -0.0003 \\
\hline 0.84 & 0.000029 & 0.000029 & & 0.274579 & 0.274578 & 0.0004 \\
\hline 0.86 & 0.000115 & 0.000115 & & 0.234832 & 0.234830 & 0.0009 \\
\hline 0.88 & 0.000387 & 0.000385 & 0.5195 & 0.197081 & 0.197077 & 0.0020 \\
\hline 0.90 & 0.001109 & 0.001105 & 0.3620 & 0.161470 & 0.161464 & 0.0037 \\
\hline 0.92 & 0.002753 & 0.002746 & 0.2549 & 0.128360 & 0.128351 & 0.0070 \\
\hline 0.94 & 0.006003 & 0.005992 & 0.1836 & 0.098336 & 0.098322 & 0.0142 \\
\hline 0.96 & 0.011655 & 0.011639 & 0.1375 & 0.072100 & 0.072081 & 0.0264 \\
\hline 0.98 & 0.020408 & 0.020390 & 0.0883 & 0.050267 & 0.050245 & 0.0438 \\
\hline 1.00 & 0.032643 & 0.032624 & 0.0582 & 0.033139 & 0.033116 & 0.0695 \\
\hline 1.02 & 0.048284 & 0.048267 & 0.0352 & 0.020568 & 0.020547 & 0.1022 \\
\hline 1.04 & 0.066823 & 0.066811 & 0.0180 & 0.011981 & 0.011964 & 0.1421 \\
\hline 1.06 & 0.087482 & 0.087475 & 0.0080 & 0.006538 & 0.006525 & 0.1992 \\
\hline 1.08 & 0.109419 & 0.109416 & 0.0027 & 0.003339 & 0.003330 & 0.2703 \\
\hline 1.10 & 0.131898 & 0.131898 & 0.0000 & 0.001596 & 0.001590 & 0.3774 \\
\hline 1.12 & 0.154374 & 0.154377 & -0.0019 & 0.000714 & 0.000711 & 0.4219 \\
\hline 1.14 & 0.176497 & 0.176502 & -0.0028 & 0.000300 & 0.000298 & 0.6711 \\
\hline 1.16 & 0.198075 & 0.198082 & -0.0035 & 0.000118 & 0.000117 & 0.8547 \\
\hline 1.18 & 0.219023 & 0.219031 & -0.0037 & 0.000044 & 0.000043 & 2.3256 \\
\hline 1.20 & 0.239317 & 0.239324 & -0.0029 & 0.000015 & 0.000015 & \\
\hline 1.22 & 0.258962 & 0.258970 & -0.0031 & 0.000005 & 0.000005 & \\
\hline 1.24 & 0.277980 & 0.277988 & -0.0029 & 0.000002 & 0.000002 & \\
\hline 1.26 & 0.296397 & 0.296405 & -0.0027 & 0.000000 & 0.000000 & \\
\hline 1.28 & 0.314238 & 0.314247 & -0.0029 & 0.000000 & 0.000000 & \\
\hline 1.30 & 0.331531 & 0.331540 & -0.0027 & 0.000000 & 0.000000 & \\
\hline
\end{tabular}

$S_{t}$ is the exchange rate at initial time $t, X$ is the exercise price, Call $O$ and $\mathrm{Put}_{\mathrm{O}}$ are call and put prices for our model, Call $\mathrm{GK}_{\mathrm{G}}$ and Put $\mathrm{GK}_{\mathrm{K}}$ are call and put prices for the G-K model, Bias $c=100\left(\right.$ Call $\left._{\mathrm{O}}-\mathrm{Call}_{\mathrm{GK}}\right) / \mathrm{Call}_{\mathrm{GK}}$, and Bias $p=100\left(\mathrm{Put}_{\mathrm{O}}-\mathrm{Put}_{\mathrm{GK}}\right) / \mathrm{Put}_{\mathrm{GK}}$.

the G-K model (36) by setting the initial time $t=1$ January 2010 when $r_{d}(t)=0.0008, r_{f}(t)=0.0049$, and $S_{t}=1.4389$. In addition, employing our FX option model as a yardstick, we examine whether or not the G-K model values correctly FX call and put options for different values of $S_{t} / X$, where $S_{t}$ is the exchange rate on 1 January 2010 and $X$ is the exercise price. Given that $S_{t}$ is fixed at 1.4389 , we vary $X$ such that $S_{t} / X$ ranges from 0.70 to 1.30 - a range large enough to include even extreme values of $X$ not commonly used in practice. Tables 1, 2, 3, and 4 show the FX option prices and pricing biases in the G-K model when time to maturity $T$ is $1,3,6$, and 9 months, respectively.

We first focus on call options. For $T=1,3,6$, and 9 months, call prices increase as $S_{t} / X$ increases from 0.70 to 1.30 under both our model and the G-K model. For example, in Table 1 where $T=1$ month, call price increases from 0.001109 to 0.032643 and to 0.131898 under our model, and from 0.001105 to 0.032624 and to 0.131898 under the G-K model as $S_{t} / X$ increases from 0.90 to 1.00 and to 1.10 , respectively. For each of the four Ts, the G-K model incorrectly values FX calls for different values of $S_{t} / X$. Specifically, it undervalues calls when $S_{t} / X$ ranges from 0.70 to 1.08 , and it overvalues calls when $S_{t} / X$ ranges from 1.18 to 1.30 . For example, in Table 2 where $T=3$ months, call price is 0.000666 under our model and 0.000657 under the G-K model for $S_{t} / X=0.80$, which amounts to a positive pricing bias of $1.3699 \%$, whereas call price is 0.330616 under our model and 0.330678 under the G$\mathrm{K}$ model for $S_{t} / X=1.30$, which amounts to a negative pricing bias of $-0.0187 \%$.

The situation is completely opposite for put options. For $T=1,3,6$, and 9 months, put prices decrease as $S_{t} / X$ increases from 0.70 to 1.30 under both models. For example, in Table 3 where $T=6$ months, put price decreases from 0.367578 to 0.082131 and to 0.008767 under our model, and from 0.367695 to 0.081844 and to 0.008639 under the G-K model as $S_{t} / X$ increases from 0.80 to 1.00 
TABLE 2: Prices for European call and put FX options when time to maturity $T=3$ months.

\begin{tabular}{|c|c|c|c|c|c|c|}
\hline$S_{t} / X$ & $\mathrm{Call}_{\mathrm{O}}$ & Call $_{\mathrm{GK}}$ & $\operatorname{Bias}_{c}$ & $\mathrm{Put}_{\mathrm{O}}$ & $\mathrm{Put}_{\mathrm{GK}}$ & Bias $_{p}$ \\
\hline 0.70 & 0.000007 & 0.000007 & & 0.617944 & 0.618028 & -0.0136 \\
\hline 0.72 & 0.000020 & 0.000020 & & 0.560879 & 0.560954 & -0.0134 \\
\hline 0.74 & 0.000055 & 0.000054 & 1.8519 & 0.506920 & 0.506986 & -0.0130 \\
\hline 0.76 & 0.000137 & 0.000135 & 1.4815 & 0.455851 & 0.455907 & -0.0123 \\
\hline 0.78 & 0.000314 & 0.000309 & 1.6181 & 0.407499 & 0.407545 & -0.0113 \\
\hline 0.80 & 0.000666 & 0.000657 & 1.3699 & 0.361749 & 0.361783 & -0.0094 \\
\hline 0.82 & 0.001315 & 0.001299 & 1.2317 & 0.318545 & 0.318566 & -0.0066 \\
\hline 0.84 & 0.002432 & 0.002407 & 1.0386 & 0.277897 & 0.277902 & -0.0018 \\
\hline 0.86 & 0.004236 & 0.004200 & 0.8571 & 0.239879 & 0.239867 & 0.0050 \\
\hline 0.88 & 0.006985 & 0.006935 & 0.7210 & 0.204615 & 0.204584 & 0.0151 \\
\hline 0.90 & 0.010951 & 0.010888 & 0.5786 & 0.172258 & 0.172208 & 0.0290 \\
\hline 0.92 & 0.016399 & 0.016322 & 0.4718 & 0.142962 & 0.142892 & 0.0490 \\
\hline 0.94 & 0.023547 & 0.023459 & 0.3751 & 0.116845 & 0.116759 & 0.0737 \\
\hline 0.96 & 0.032548 & 0.032452 & 0.2958 & 0.093967 & 0.093868 & 0.1055 \\
\hline 0.98 & 0.043461 & 0.043362 & 0.2283 & 0.074302 & 0.074195 & 0.1442 \\
\hline 1.00 & 0.056252 & 0.056155 & 0.1727 & 0.057739 & 0.057629 & 0.1909 \\
\hline 1.02 & 0.070794 & 0.070703 & 0.1287 & 0.044077 & 0.043969 & 0.2456 \\
\hline 1.04 & 0.086884 & 0.086803 & 0.0933 & 0.033048 & 0.032946 & 0.3096 \\
\hline 1.06 & 0.104267 & 0.104200 & 0.0643 & 0.024335 & 0.024243 & 0.3795 \\
\hline 1.08 & 0.122661 & 0.122610 & 0.0416 & 0.017600 & 0.017520 & 0.4566 \\
\hline 1.10 & 0.141781 & 0.141745 & 0.0254 & 0.012505 & 0.012436 & 0.5548 \\
\hline 1.12 & 0.161356 & 0.161337 & 0.0118 & 0.008730 & 0.008674 & 0.6456 \\
\hline 1.14 & 0.181148 & 0.181143 & 0.0028 & 0.005991 & 0.005946 & 0.7568 \\
\hline 1.16 & 0.200954 & 0.200963 & -0.0045 & 0.004042 & 0.004007 & 0.8735 \\
\hline 1.18 & 0.220612 & 0.220632 & -0.0091 & 0.002683 & 0.002657 & 0.9785 \\
\hline 1.20 & 0.239997 & 0.240028 & -0.0129 & 0.001753 & 0.001733 & 1.1541 \\
\hline 1.22 & 0.259022 & 0.259062 & -0.0154 & 0.001128 & 0.001114 & 1.2567 \\
\hline 1.24 & 0.277625 & 0.277672 & -0.0169 & 0.000715 & 0.000705 & 1.4184 \\
\hline 1.26 & 0.295770 & 0.295822 & -0.0176 & 0.000446 & 0.000440 & 1.3636 \\
\hline 1.28 & 0.313436 & 0.313493 & -0.0182 & 0.000275 & 0.000270 & 1.8519 \\
\hline 1.30 & 0.330616 & 0.330678 & -0.0187 & 0.000167 & 0.000164 & 1.8293 \\
\hline
\end{tabular}

$S_{t}$ is the exchange rate at initial time $t, X$ is the exercise price, Call $O$ and Put ${ }_{O}$ are call and put prices for our model, Call ${ }_{\mathrm{GK}}$ and Put ${ }_{\mathrm{GK}}$ are call and put prices for the G-K model, $\operatorname{Bias}_{c}=100\left(\right.$ Call $_{O}-$ Call $\left._{\mathrm{GK}}\right) / \mathrm{Call}_{\mathrm{GK}}$, and $\mathrm{Bias}_{p}=100\left(\mathrm{Put}_{\mathrm{O}}-\mathrm{Put}_{\mathrm{GK}}\right) / \mathrm{Put}_{\mathrm{GK}}$.

and to 1.20, respectively. For each of the four Ts, the G-K model incorrectly values FX puts for different values of $S_{t} / X$. Specifically, it overvalues puts when $S_{t} / X$ ranges from 0.70 to 0.82 , and it undervalues puts when $S_{t} / X$ ranges from 0.86 to 1.30. For example, in Table 4 where $T=9$ months, put price is 0.375783 under our model and 0.376021 under the G-K model for $S_{t} / X=0.80$, which amounts to a negative pricing bias of $-0.0633 \%$, whereas put price is 0.006367 under our model and 0.006198 under the G-K model for $S_{t} / X=1.30$, which amounts to a positive pricing bias of $2.7267 \%$.

Foreign exchange options are actively traded on the Philadelphia Stock Exchange. The contract size of a eurocurrency option is $€ 62,500$. For example, when $T=9$ months and $S_{t} / X=0.90$, call premium is $(\$ 0.042077$ $\times 62,500)=\$ 2,629.81$ under our model and $(\$ 0.041614 \times$ $62,500)=\$ 2,600.88$ under the G-K model-a difference of $\$ 28.93$ underpaid by a call option buyer; similarly, when $T=$ 9 months and $S_{t} / X=1.10$, put premium is $(\$ 0.044294$ $\times 62,500)=\$ 2,768.38$ under our model and $(\$ 0.043837 \times$ $62,500)=\$ 2,739.81$ under the G-K model-a difference of $\$ 28.57$ underpaid by a put option buyer. In other words, option sellers are at an obvious disadvantage if FX option valuation is based on the G-K model.

\section{Conclusion}

Prior research often assumes constant spot rates when valuing FX options. The traditional G-K FX option model assumes that both domestic and foreign spot rates remain unchanged over the life of the FX option. The fact of the matter is that both spot rates are changing continuously and stochastically through time. In this paper, we utilize stochastic calculus to develop a continuous-time model for valuing European call and put options on foreign exchange when both spot rates are assumed to follow a generalized Wiener process. Using the dollar/euro exchange rate as input for parameter estimation 
TABLE 3: Prices for European call and put FX options when time to maturity $T=6$ months.

\begin{tabular}{|c|c|c|c|c|c|c|}
\hline$S_{t} / X$ & Call $_{O}$ & Call $_{\mathrm{GK}}$ & $\operatorname{Bias}_{c}$ & $\mathrm{Put}_{\mathrm{O}}$ & $\mathrm{Put}_{\mathrm{GK}}$ & Bias $_{p}$ \\
\hline 0.70 & 0.000418 & 0.000404 & 3.4653 & 0.619406 & 0.619774 & -0.0594 \\
\hline 0.72 & 0.000760 & 0.000736 & 3.2609 & 0.562709 & 0.563030 & -0.0570 \\
\hline 0.74 & 0.001314 & 0.001278 & 2.8169 & 0.509307 & 0.509581 & -0.0538 \\
\hline 0.76 & 0.002172 & 0.002120 & 2.4528 & 0.459050 & 0.459273 & -0.0486 \\
\hline 0.78 & 0.003447 & 0.003374 & 2.1636 & 0.411830 & 0.412001 & -0.0415 \\
\hline 0.80 & 0.005265 & 0.005168 & 1.8769 & 0.367578 & 0.367695 & -0.0318 \\
\hline 0.82 & 0.007767 & 0.007643 & 1.6224 & 0.326258 & 0.326318 & -0.0184 \\
\hline 0.84 & 0.011095 & 0.010942 & 1.3983 & 0.287850 & 0.287854 & -0.0014 \\
\hline 0.86 & 0.015386 & 0.015204 & 1.1971 & 0.252347 & 0.252296 & 0.0202 \\
\hline 0.88 & 0.020765 & 0.020555 & 1.0216 & 0.219740 & 0.219636 & 0.0474 \\
\hline 0.90 & 0.027330 & 0.027095 & 0.8673 & 0.190008 & 0.189855 & 0.0806 \\
\hline 0.92 & 0.035151 & 0.034897 & 0.7279 & 0.163110 & 0.162915 & 0.1197 \\
\hline 0.94 & 0.044264 & 0.043997 & 0.6069 & 0.138981 & 0.138750 & 0.1665 \\
\hline 0.96 & 0.054668 & 0.054394 & 0.5037 & 0.117527 & 0.117270 & 0.2192 \\
\hline 0.98 & 0.066323 & 0.066051 & 0.4118 & 0.098627 & 0.098350 & 0.2816 \\
\hline 1.00 & 0.079161 & 0.078898 & 0.3333 & 0.082131 & 0.081844 & 0.3507 \\
\hline 1.02 & 0.093084 & 0.092837 & 0.2661 & 0.067869 & 0.067580 & 0.4276 \\
\hline 1.04 & 0.107972 & 0.107748 & 0.2079 & 0.055657 & 0.055373 & 0.5129 \\
\hline 1.06 & 0.123689 & 0.123494 & 0.1579 & 0.045298 & 0.045025 & 0.6063 \\
\hline 1.08 & 0.140095 & 0.139932 & 0.1165 & 0.036592 & 0.036335 & 0.7073 \\
\hline 1.10 & 0.157045 & 0.156917 & 0.0816 & 0.029344 & 0.029106 & 0.8177 \\
\hline 1.12 & 0.174398 & 0.174306 & 0.0528 & 0.023362 & 0.023146 & 0.9332 \\
\hline 1.14 & 0.192021 & 0.191967 & 0.0281 & 0.018470 & 0.018276 & 1.0615 \\
\hline 1.16 & 0.209793 & 0.209776 & 0.0081 & 0.014503 & 0.014332 & 1.1931 \\
\hline 1.18 & 0.227604 & 0.227623 & -0.0083 & 0.011312 & 0.011163 & 1.3348 \\
\hline 1.20 & 0.245361 & 0.245414 & -0.0216 & 0.008767 & 0.008639 & 1.4817 \\
\hline 1.22 & 0.262982 & 0.263067 & -0.0323 & 0.006752 & 0.006643 & 1.6408 \\
\hline 1.24 & 0.280401 & 0.280517 & -0.0414 & 0.005169 & 0.005077 & 1.8121 \\
\hline 1.26 & 0.297566 & 0.297709 & -0.0480 & 0.003934 & 0.003857 & 1.9964 \\
\hline 1.28 & 0.314434 & 0.314602 & -0.0534 & 0.002977 & 0.002914 & 2.1620 \\
\hline 1.30 & 0.330974 & 0.331165 & -0.0577 & 0.002241 & 0.002189 & 2.3755 \\
\hline
\end{tabular}

$S_{t}$ is the exchange rate at initial time $t, X$ is the exercise price, Call $O$ and Put $O$ are call and put prices for our model, Call ${ }_{\mathrm{GK}}$ and Put ${ }_{\mathrm{GK}}$ are call and put prices for the G-K model, Bias $=100\left(\right.$ Call $_{O}-$ Call $\left._{G K}\right) / C_{\text {Call }}$ GK , and Bias $p=100\left(\right.$ Put $_{O}-$ Put $\left._{G K}\right) /$ Put $_{\mathrm{GK}}$.

and employing our FX option model as a yardstick, our numerical results show that the G-K model values incorrectly both call and put options for different values of $S_{t} / X$. Specifically, it undervalues calls when $S_{t} / X$ is between 0.70 and 1.08, and it overvalues calls when $S_{t} / X$ is between 1.18 and 1.30 , whereas it overvalues puts when $S_{t} / X$ is between 0.70 and 0.82 , and it undervalues puts when $S_{t} / X$ is between 0.86 and 1.30 .

\section{Appendix}

To derive (27), we first prove the following:

$$
\begin{gathered}
\int_{0}^{\infty} \cos [k(\omega-\delta)] \exp \left[-\frac{1}{2} k^{2} V\right] d k \\
=\sqrt{\frac{\pi}{2 V}} \exp \left[-\frac{(\omega-\delta)^{2}}{2 V}\right] .
\end{gathered}
$$

Proof. Since $\cos [k(\omega-\delta)]=(1 / 2) \exp [i k(\omega-\delta)]+(1 / 2)$ $\exp [-i k(\omega-\delta)]$, we obtain

$$
\begin{aligned}
\cos [k(\omega-\delta)] \exp \left[-\frac{1}{2} k^{2} V\right] \\
=\frac{1}{2} \exp \left[-\frac{1}{2} k^{2} V+i k(\omega-\delta)\right] \\
+\frac{1}{2} \exp \left[-\frac{1}{2} k^{2} V-i k(\omega-\delta)\right],
\end{aligned}
$$

where $i=\sqrt{-1}$. Since $-(1 / 2) k^{2} V+i k(\omega-\delta)=$ $-\left((\omega-\delta)^{2} / 2 V\right)-[(k V-i(\omega-\delta)) / \sqrt{2 V}]^{2}$, we have

$$
\begin{aligned}
& \frac{1}{2} \int_{0}^{\infty} \exp \left[-\frac{1}{2} k^{2} V+i k(\omega-\delta)\right] d k \\
& \quad=\frac{1}{2} \int_{0}^{\infty} \exp \left[-\frac{(\omega-\delta)^{2}}{2 V}-\left(\frac{k V-i(\omega-\delta)}{\sqrt{2 V}}\right)^{2}\right] d k
\end{aligned}
$$


TABLE 4: Prices for European call and put FX options when time to maturity $T=9$ months.

\begin{tabular}{|c|c|c|c|c|c|c|}
\hline$S_{t} / X$ & Call $_{O}$ & Call $_{\mathrm{GK}}$ & $\operatorname{Bias}_{c}$ & $\mathrm{Put}_{\mathrm{O}}$ & $\mathrm{Put}_{\mathrm{GK}}$ & Bias $_{p}$ \\
\hline 0.70 & 0.002017 & 0.001937 & 4.1301 & 0.621839 & 0.622653 & -0.1307 \\
\hline 0.72 & 0.003077 & 0.002967 & 3.7074 & 0.565920 & 0.566619 & -0.1234 \\
\hline 0.74 & 0.004544 & 0.004401 & 3.2493 & 0.513488 & 0.514072 & -0.1136 \\
\hline 0.76 & 0.006516 & 0.006333 & 2.8896 & 0.464397 & 0.464865 & -0.1007 \\
\hline 0.78 & 0.009091 & 0.008865 & 2.5494 & 0.418528 & 0.418880 & -0.0840 \\
\hline 0.80 & 0.012367 & 0.012096 & 2.2404 & 0.375783 & 0.376021 & -0.0633 \\
\hline 0.82 & 0.016438 & 0.016122 & 1.9601 & 0.336077 & 0.336204 & -0.0378 \\
\hline 0.84 & 0.021386 & 0.021026 & 1.7122 & 0.299333 & 0.299353 & -0.0067 \\
\hline 0.86 & 0.027279 & 0.026878 & 1.4919 & 0.265473 & 0.265392 & 0.0305 \\
\hline 0.88 & 0.034166 & 0.033730 & 1.2926 & 0.234414 & 0.234242 & 0.0734 \\
\hline 0.90 & 0.042077 & 0.041614 & 1.1126 & 0.206066 & 0.205811 & 0.1239 \\
\hline 0.92 & 0.051021 & 0.050540 & 0.9517 & 0.180327 & 0.180002 & 0.1806 \\
\hline 0.94 & 0.060985 & 0.060494 & 0.8117 & 0.157083 & 0.156699 & 0.2451 \\
\hline 0.96 & 0.071935 & 0.071446 & 0.6844 & 0.136210 & 0.135780 & 0.3167 \\
\hline 0.98 & 0.083822 & 0.083344 & 0.5735 & 0.117572 & 0.117107 & 0.3971 \\
\hline 1.00 & 0.096577 & 0.096121 & 0.4744 & 0.101024 & 0.100537 & 0.4844 \\
\hline 1.02 & 0.110123 & 0.109698 & 0.3874 & 0.086416 & 0.085917 & 0.5808 \\
\hline 1.04 & 0.124372 & 0.123986 & 0.3113 & 0.073592 & 0.073092 & 0.6841 \\
\hline 1.06 & 0.139229 & 0.138890 & 0.2441 & 0.062399 & 0.061907 & 0.7947 \\
\hline 1.08 & 0.154598 & 0.154311 & 0.1860 & 0.052683 & 0.052205 & 0.9156 \\
\hline 1.10 & 0.170382 & 0.170153 & 0.1346 & 0.044294 & 0.043837 & 1.0425 \\
\hline 1.12 & 0.186487 & 0.186318 & 0.0907 & 0.037090 & 0.036658 & 1.1785 \\
\hline 1.14 & 0.202823 & 0.202717 & 0.0523 & 0.030934 & 0.030531 & 1.3200 \\
\hline 1.16 & 0.219306 & 0.219263 & 0.0196 & 0.025701 & 0.025328 & 1.4727 \\
\hline 1.18 & 0.235858 & 0.235878 & -0.0085 & 0.021273 & 0.020932 & 1.6291 \\
\hline 1.20 & 0.252410 & 0.252493 & -0.0329 & 0.017544 & 0.017235 & 1.7929 \\
\hline 1.22 & 0.268900 & 0.269044 & -0.0535 & 0.014419 & 0.014141 & 1.9659 \\
\hline 1.24 & 0.285275 & 0.285477 & -0.0708 & 0.011810 & 0.011562 & 2.1450 \\
\hline 1.26 & 0.301487 & 0.301745 & -0.0855 & 0.009642 & 0.009422 & 2.3350 \\
\hline 1.28 & 0.317498 & 0.317809 & -0.0979 & 0.007847 & 0.007654 & 2.5216 \\
\hline 1.30 & 0.333276 & 0.333638 & -0.1085 & 0.006367 & 0.006198 & 2.7267 \\
\hline
\end{tabular}

$S_{t}$ is the exchange rate at initial time $t, X$ is the exercise price, Call ${ }_{\mathrm{O}}$ and $\mathrm{Put}_{\mathrm{O}}$ are call and put prices for our model, Call ${ }_{\mathrm{GK}}$ and Put ${ }_{\mathrm{GK}}$ are call and put prices for the G-K model, $\operatorname{Bias}_{c}=100\left(\right.$ Call $_{O}-$ Call $\left._{G K}\right) / \mathrm{Call}_{\mathrm{GK}}$, and $\mathrm{Bias}_{p}=100\left(\mathrm{Put}_{\mathrm{O}}-\mathrm{Put}_{\mathrm{GK}}\right) / \mathrm{Put}_{\mathrm{GK}}$.

$$
\begin{aligned}
= & \frac{1}{2} \exp \left[-\frac{(\omega-\delta)^{2}}{2 V}\right] & =\frac{1}{2} \sqrt{\frac{\pi}{2 V}} \exp \left[-\frac{(\omega-\delta)^{2}}{2 V}\right] . \\
& \times \int_{0}^{\infty} \exp \left[-\left(\frac{k V-i(\omega-\delta)}{\sqrt{2 V}}\right)^{2}\right] d k . &
\end{aligned}
$$

(A.3)

Similarly, since $-(1 / 2) k^{2} V-i k(\omega-\delta)=-\left((\omega-\delta)^{2} / 2 V\right)-$ $[(k V+i(\omega-\delta)) / \sqrt{2 V}]^{2}$, we have

Letting $x=(k V-i(\omega-\delta)) / \sqrt{2 V}$, which implies $d k=$ $\sqrt{(2 / V)} d x$, we have

$$
\begin{aligned}
\frac{1}{2} \int_{0}^{\infty} \exp \left[-\frac{1}{2} k^{2} V-i k(\omega-\delta)\right] d k \\
=\frac{1}{2} \exp \left[-\frac{(\omega-\delta)^{2}}{2 V}\right] \\
\quad \times \int_{0}^{\infty} \exp \left[-\left(\frac{k V+i(\omega-\delta)}{\sqrt{2 V}}\right)^{2}\right] d k .
\end{aligned}
$$$$
\frac{1}{2} \int_{0}^{\infty} \exp \left[-\frac{1}{2} k^{2} V+i k(\omega-\delta)\right] d k
$$$$
=\frac{1}{2} \exp \left[-\frac{(\omega-\delta)^{2}}{2 V}\right] \int_{0}^{\infty} \exp \left[-x^{2}\right] \sqrt{\frac{2}{V}} d x
$$ 
Letting $z=(k V+i(\omega-\delta)) / \sqrt{2 V}$, which implies $d k=$ $\sqrt{(2 / V)} d z$, we have

$$
\begin{aligned}
\frac{1}{2} \int_{0}^{\infty} & \exp \left[-\frac{1}{2} k^{2} V-i k(\omega-\delta)\right] d k \\
\quad= & \frac{1}{2} \exp \left[-\frac{(\omega-\delta)^{2}}{2 V}\right] \int_{0}^{\infty} \exp \left[-z^{2}\right] \sqrt{\frac{2}{V}} d z \\
\quad= & \frac{1}{2} \sqrt{\frac{\pi}{2 V}} \exp \left[-\frac{(\omega-\delta)^{2}}{2 V}\right] .
\end{aligned}
$$

Hence, combining (A.4) and (A.6), we obtain (A.1). Now substituting $A(k)=(1 / \pi) \int_{-\infty}^{\infty} f(\omega) \cos (k \omega) d \omega$ and $B(k)=$ $(1 / \pi) \int_{-\infty}^{\infty} f(\omega) \sin (k \omega) d \omega$ into (26), we obtain

$$
\begin{gathered}
u(\delta, V)=\frac{1}{\pi} \int_{0}^{\infty}\left\{\int_{-\infty}^{\infty}[\cos (k \omega) \cos (k \delta)+\sin (k \omega) \sin (k \delta)]\right. \\
\times f(\omega) d \omega\} \exp \left(-\frac{1}{2} k^{2} V\right) d k \\
=\frac{1}{\pi} \int_{-\infty}^{\infty} f(\omega) \int_{0}^{\infty} \cos [k(\omega-\delta)] \\
\times \exp \left(-\frac{1}{2} k^{2} V\right) d k d \omega .
\end{gathered}
$$

Substituting (A.1) into (A.7), we get (27) as follows:

$$
\begin{aligned}
u(\delta, V) & =\frac{1}{\pi} \int_{-\infty}^{\infty} f(\omega)\left[\sqrt{\frac{\pi}{2 V}} \exp \left(-\frac{(\omega-\delta)^{2}}{2 V}\right)\right] d \omega \\
& =\frac{1}{\sqrt{2 V \pi}} \int_{-\infty}^{\infty} f(\omega) \exp \left(-\frac{(\omega-\delta)^{2}}{2 V}\right) d \omega
\end{aligned}
$$

\section{Acknowledgments}

The author would like to thank two anonymous referees whose valuable suggestions have greatly improved the quality of this paper. This research was supported by the National Science Council of Taiwan (ROC) under Grant NSC-98-2410H-130-028.

\section{References}

[1] R. C. Merton, Continuous-Time Finance, Blackwell, Hoboken, NJ, USA, 1990.

[2] I. Karatzas and S. E. Shreve, Brownian Motion and Stochastic Calculus, vol. 113 of Graduate Texts in Mathematics, Springer, New York, NY, USA, 2nd edition, 1991.

[3] S. E. Shreve, Stochastic Calculus for Finance. II. Continuous-Time Models, Springer Finance, Springer, New York, NY, USA, 2004.

[4] F. Black and M. Scholes, "The pricing of options and corporate liabilities," Journal of Political Economy, vol. 81, pp. 637-659, 1973.
[5] M. B. Garman and S. W. Kohlhagen, "Foreign currency option values," Journal of International Money and Finance, vol. 2, no. 3, pp. 231-237, 1983.

[6] J. C. Hull, Options, Futures, and Other Derivatives, PrenticeHall, Upper Saddle River, NJ, USA, 6th edition, 2006.

[7] T. S. Ho, R. C. Stapleton, and M. G. Subrahmanyam, "The valuation of American options with stochastic interest rates: a generalization of the Geske-Johnson technique," Journal of Finance, vol. 52, no. 2, pp. 827-840, 1997.

[8] K. Zhang and S. Wang, "Pricing American bond options using a penalty method," Automatica, vol. 48, no. 3, pp. 472-479, 2012.

[9] S. Wang, X. Q. Yang, and K. L. Teo, "Power penalty method for a linear complementarity problem arising from American option valuation," Journal of Optimization Theory and Applications, vol. 129, no. 2, pp. 227-254, 2006.

[10] K. Zhang, X. Yang, and K. L. Teo, "Convergence analysis of a monotonic penalty method for American option pricing," Journal of Mathematical Analysis and Applications, vol. 348, no. 2, pp. 915-926, 2008.

[11] L. Arnold, Stochastic Differential Equations: Theory and Applications, John Wiley \& Sons, New York, NY, USA, 1974.

[12] B. Øksendal, Stochastic Differential Equations. An Introduction with Applications, Universitext, Springer, Berlin, Germany, 5th edition, 1998.

[13] P. Wilmott, Derivatives: The Theory and Practice of Financial Engineering, John Wiley \& Sons, New York, NY, USA, 1998.

[14] N. H. Asmar, Partial Differential Equations with Fourier Series and Boundary Value Problems, Pearson Prentice Hall, Upper Saddle River, NJ, USA, 2nd edition, 2005.

[15] J. W. Brown and R. V. Churchill, Fourier Series and Boundary Value Problems, McGraw-Hill, New York, NY, USA, 5th edition, 1993.

[16] M. Humi and W. B. Miller, Boundary Value Problems and Partial Differential Equations, PWS-Kent, Boston, Mass, USA, 1992.

[17] W. Margrabe, "The value of an option to exchange one asset for another," Journal of Finance, vol. 33, pp. 177-186, 1978.

[18] R. C. Merton, "Theory of rational option pricing," Bell Journal of Economics \& Management Science, vol. 4, pp. 141-183, 1973.

[19] C. W. Smith Jr., "Option pricing. A review," Journal of Financial Economics, vol. 3, no. 1-2, pp. 3-51, 1976. 


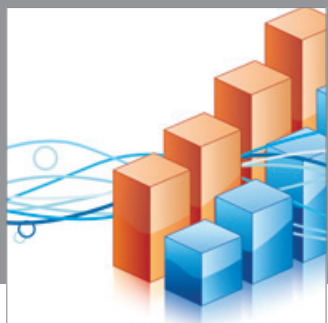

Advances in

Operations Research

mansans

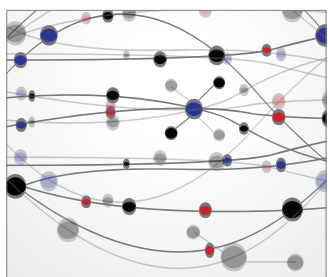

The Scientific World Journal
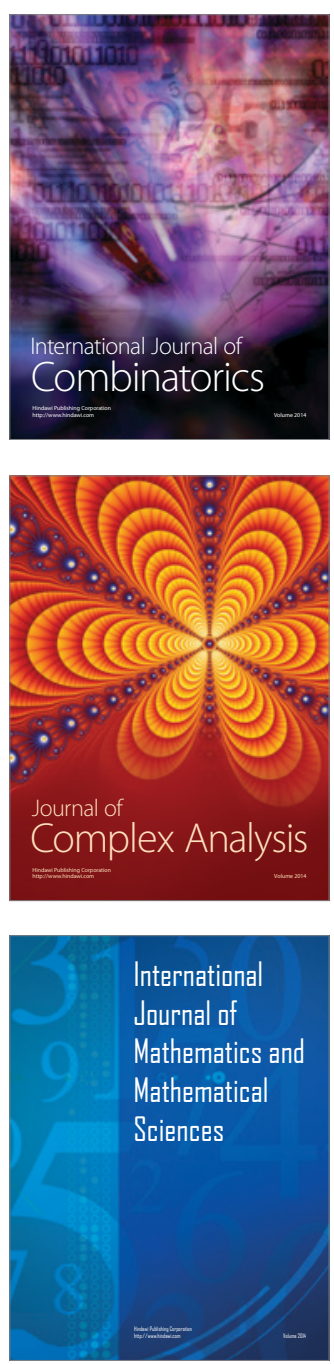
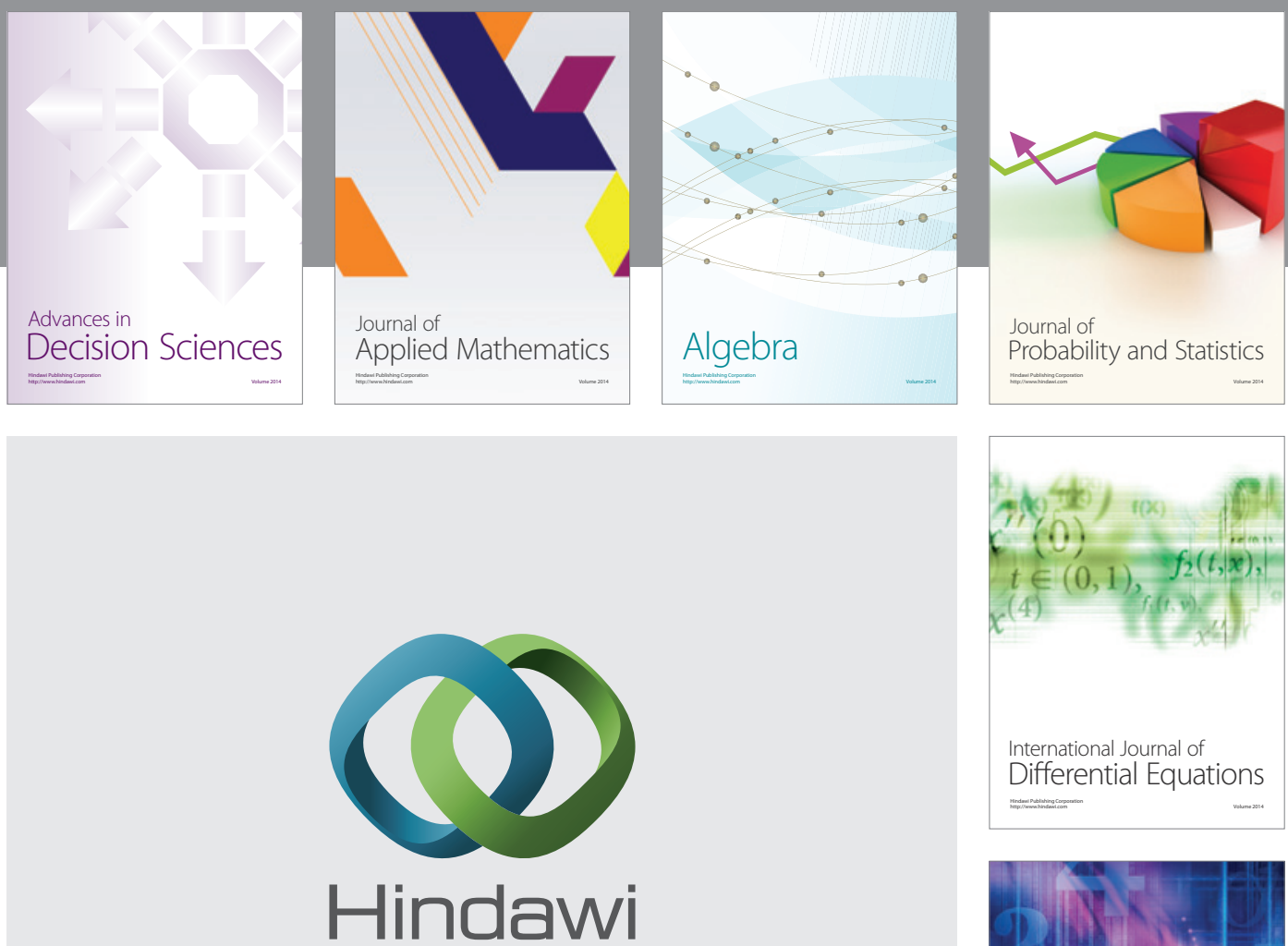

Submit your manuscripts at http://www.hindawi.com
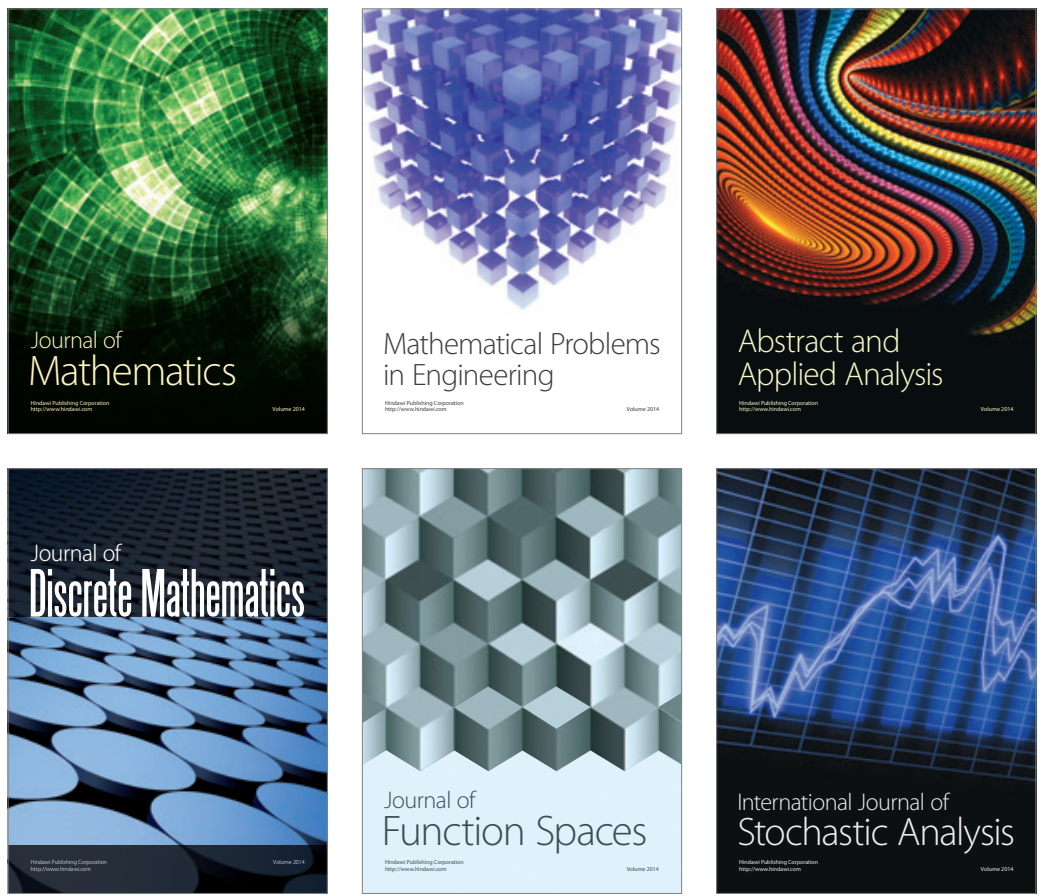

Journal of

Function Spaces

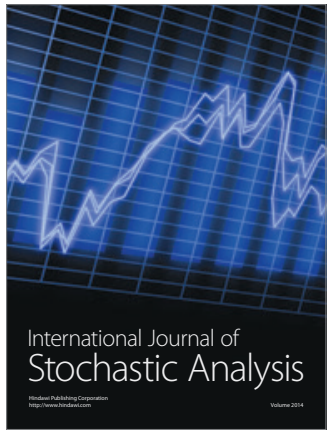

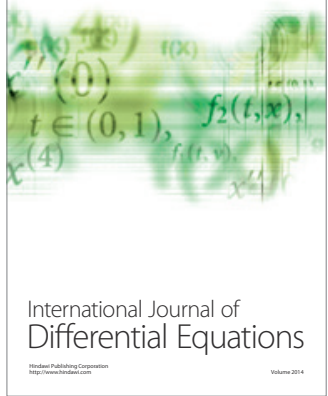
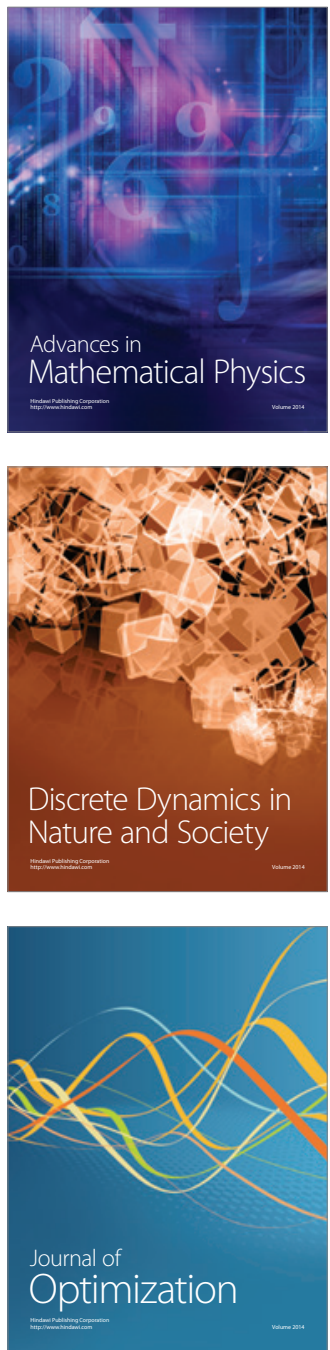